\title{
Generalized Courant-Snyder theory for charged particle dynamics in general focusing lattices
}

\author{
Hong Qin, ${ }^{1,2}$ Ronald C. Davidson, ${ }^{1}$ Moses Chung, ${ }^{3}$ and Joshua W. Burby ${ }^{1}$ \\ ${ }^{1}$ Plasma Physics Laboratory, Princeton University, Princeton, NJ 08543 \\ ${ }^{2}$ Department of Modern Physics, University of Science \\ and Technology of China, Hefei, Anhui 230026, China \\ ${ }^{3}$ Accelerator Physics Center, Fermi National Accelerator Laboratory, Batavia, IL 60510

\begin{abstract}
Courant-Snyder (CS) theory for one degree of freedom is generalized to the case of coupled transverse dynamics in general linear focusing lattices with quadrupole, skew-quadrupole, dipole, and solenoidal components, as well as torsion of the fiducial orbit and variation of beam energy. The envelope function is generalized into an envelope matrix, and the phase advance is generalized into a $4 \mathrm{D}$ sympletic rotation. The envelope equation, transfer matrix, and the CS invariant of the original CS theory, all have their counterparts, with remarkably similar expressions, in the generalized theory.
\end{abstract}

PACS numbers: 29.27.-a,52.20.Dq 
For a charged particle in an uncoupled quadrupole focusing lattice, the classical CourantSnyder (CS) theory [1] gives a complete description of its dynamics. Because the dynamics in the $x$-direction and $y$-direction are decoupled, the equations of motion in both directions are given by

$$
\ddot{q}+\kappa_{q}(t) q=0,
$$

where $q$ represents either of the transverse coordinates, $x$ or $y$, and $\kappa_{q}(t)$ is the timedependent linear focusing strength. For a quadrupole lattice, $\kappa_{x}(t)=-\kappa_{y}(t)$. The CS theory states that the solution of Eq. (1) can be expressed as a time-dependent symplectic map applied to the initial phase space coordinates, i.e., $(q, \dot{q})^{T}=M_{1}(t)\left(q_{0}, \dot{q}_{0}\right)^{T}$,

$$
M_{1}(t)=\left(\begin{array}{cc}
\sqrt{\frac{\beta}{\beta_{0}}}\left[\cos \phi+\alpha_{0} \sin \phi\right] & \sqrt{\beta \beta_{0}} \sin \phi \\
-\frac{1+\alpha \alpha_{0}}{\sqrt{\beta \beta_{0}}} \sin \phi+\frac{\alpha_{0}-\alpha}{\sqrt{\beta \beta_{0}}} \cos \phi \sqrt{\frac{\beta_{0}}{\beta}}[\cos \phi-\alpha \sin \phi]
\end{array}\right),
$$

where the superscript "T" denotes the transpose operation, and $\alpha(t), \beta(t)$, and $\phi(t)$ are time-dependent functions defined in terms of the envelope function $w(t)$ by

$$
\beta(t) \equiv w^{2}(t), \alpha(t) \equiv-w \dot{w}, \phi(t) \equiv \int_{0}^{t} \frac{d t}{\beta(t)} .
$$

The envelope function $w(t)$ is determined by the nonlinear envelope equation

$$
\ddot{w}+\kappa_{q}(t) w=w^{-3} .
$$

In Eq. (2) $q_{0}=q(t=0), \dot{q}_{0}=\dot{q}(t=0), \beta_{0}=\beta(t=0)$, and $\alpha_{0}=\alpha(t=0)$ are initial conditions. The transfer matrix $M_{1}(t)$ can be decomposed as [2]

$$
M_{1}(t)=\left(\begin{array}{cc}
w & 0 \\
\dot{w} & \frac{1}{w}
\end{array}\right)\left(\begin{array}{cc}
\cos \phi & \sin \phi \\
-\sin \phi & \cos \phi
\end{array}\right)\left(\begin{array}{cc}
w_{0}^{-1} & 0 \\
-\dot{w}_{0} & w_{0}
\end{array}\right),
$$

from which it is clear that the physical meaning of $\phi$ is the phase advance, and $\beta^{-1}=w^{-2}$ is the rate of phase advance. The Courant-Snyder invariant $[1,3]$ is given by

$$
\begin{gathered}
I=\frac{q^{2}}{w^{2}}+(w \dot{q}-\dot{w} q)^{2}=(q, \dot{q})\left(\begin{array}{cc}
\gamma & \alpha \\
\alpha & \beta
\end{array}\right)\left(\begin{array}{l}
q \\
\dot{q}
\end{array}\right), \\
\gamma(t) \equiv w^{-2}+\dot{w}^{2}
\end{gathered}
$$

The functions $\alpha(t), \beta(t)$, and $\gamma(t)$ defined by Eqs. (3) and (7) are known as the Twiss parameters. These main results of the CS theory constitute a parameterization of the 
symplectic transfer map $M_{1}(t)$ for a standard uncoupled quadrupole lattice, and provide the foundation for accelerator and beam physics. Mathematically, there are different schemes to parameterize a time-dependent symplectic map. Among these, the CS parameterization is unique because it describes the physics of charged particle dynamics. The main components of the CS theory, i.e., the phase advance, the envelope equation, the transfer matrix, and the CS invariant are of considerable importance to beam physics. The envelope function describes the transverse dimensions in configuration space while the CS invariant defines the emittance in phase space. This theoretical framework also makes it possible to investigate collective effects associated with high-intensity beams. For instance, it is used to construct the Kapchinskij-Vladimirskij distribution [4-6].

However, the CS theory can only be applied to the ideal case of an uncoupled quadrupole focusing lattices. In realistic accelerators, there also exist bending magnets, torsion of the design orbit (fiducial orbit), and skew-quadrupole components introduced intentionally or by misalignment. In certain applications, such as the NDCX-II experiment [7], solenoidal magnets are also used. When these additional linear components are included, the transverse dynamics are coupled, and the dynamics of a charged particle relative to the fiducial orbit are governed by a general time dependent Hamiltonian [8]

$$
H=\frac{1}{2} z^{T} A z, \quad A=\left(\begin{array}{cc}
\kappa(t) & R(t) \\
R(t)^{T} & m^{-1}(t)
\end{array}\right) .
$$

Here, $z=\left(x, y, p_{x}, p_{y}\right)^{T}$ are the transverse phase space coordinates, and $\kappa(t), R(t)$, and $m^{-1}(t)$ are time-dependent $2 \times 2$ matrices. The matrices $\kappa(t)$ and $m^{-1}(t)$ are also symmetric. The skew-quadrupole and dipole components are included in the off-diagonal terms of $\kappa(t)$, and the solenoidal component and the torsion of the fiducial orbit are included in $R(t)$. The variation of beam energy along the the fiducial orbit is reflected in the mass matrix $m^{-1}(t)$. For complete generality, we further allow $m^{-1}(t)$ to be any real symmetric matrix. The transfer matrix $M(t)$ corresponding to $H$ is a time-dependent $4 \times 4$ symplectic matrix, which has 10 time-dependent parameters and admits many different schemes for parameterization. Teng and Edwards [9-11] first derived a set of parameterization schemes for $M(t)$, some of which have been adopted in lattice design and particle tracking codes, such as the MAD code [12]. A class of different parameterizations has also been developed by Dattoli, et al. [13]. However, these parameterization schemes are rather mathematical and fail to connect 
with important physical paramenters of the beam, in contrast to the original CS theory. For example, they cannot be used effectively to describe the instability properties of the coupled dynamics, and are not directly related to beam envelopes and emittance. In order to describe beam envelopes for coupled dynamics, Ripken [14, 15] developed a method without using these parameterization schemes. In short, the elegant and much-needed connection with the physics of beam dynamics in the original CS theory for 1-degree of freedom is lost in these parameterization schemes.

Recently we have generalized the CS theory for uncoupled dynamics to treat coupled dynamics induced by a skew-quadrupole lattice [16-19]. In this Letter, we complete the development of the generalized CS theory by allowing the focusing lattice to assume the most general form in Eq. (8), with bending magnets, torsion of the design orbit, and solenoidal magnets, in addition to quadrupole and skew-quadrupole components. In this generalized theory, the physics components of the original CS theory, i.e., the phase advance, the envelope equation, the transfer matrix, and the CS invariant are all generalized to the 2D coupled case with identical structure.

We will use a time-dependent symplectic transformation technique $[16,19,20]$. A generalized envelope equation in $2 \times 2$ matrix form for the most general form of coupled dynamics is developed [see Eq. (16)]. In the original CS theory, the 1D envelope equation (4) plays a central role. It is also an important equation in other branches of physics, and has been discovered or re-discovered many times [21-25]. In quantum physics, it is also known as the Ermakov-Milne-Pinney equation [21-23], which has been utilized to study non-adiabatic Berry phases [26] in 1D time-dependent quantum systems [27, 28]. A brief account of the history of the 1D envelope equation can be found in Ref. [3]. The generalization of the 1D envelope equation to higher dimensions for the most general Hamiltonian is also expected to have many possible applications in areas other than beam physics. In addition, the 1D CS invariant given by Eq. (6), also known as the Lewis invariant [24, 25] in quantum physics, is generalized to higher dimension in Eq. (22).

The 1D phase advance concept is generalized to a time-dependent matrix $P$, which belongs to the symplectic rotation group $S p(4) \cap S O(4)=U(2)$. Here, $S p(4), S O(4)$, and $U(2)$ denote the groups of $4 \times 4$ symplectic matrices, $4 \times 4$ rotation matrices, and $2 \times 2$ unitary matrices, respectively.

The generalized decomposition for the symplectic map $M(t)$ is given by Eq. (21), which 
has exactly the same structure as the original 1D CS theory given by Eq. (5). In addition to its aesthetic appearance, the generalized CS theory provide an effective tool to describe the beam physics determined by the most general Hamiltonian. The $2 \times 2$ envelope matrix $w$ defines the transverse dimension of the beam, and the generalized CS invariant defines the emittance. We will also show that the stability properties of a charged particle are completely determined by the generalized phase advance $P(t)$, which is a time-dependent curve in $S p(4) \cap S O(4)=U(2)$.

The most essential part of the generalized theory is the decomposition of the timedependent symplectic coordinate transformation $G$ given by Eq. (20). The decomposition of $G$ as a product of $S P$ is the well-known Iwasawa decomposition for a semi-simple Lie group [29]. However, the unique feature of the theory developed here is that the decomposition is given from the viewpoint of dynamics and self-consistently constructed from the generalized envelope equation. It is a pleasant surprise to find the deep connection between the original CS theory for charged particle dynamics [1] and the Iwasawa decomposition for Lie groups [29], two theoretical works developed concurrently.

For the present application to beam transverse dynamics, the degree of freedom is 2 . But the theory developed is valid for any degree of freedom. For a system with $n$-degrees of freedom, the time-dependent matrix $A(t)$ specifying the Hamiltonian in Eq. (8) will be $2 n \times 2 n$, the envelope matrix will be $n \times n$, and the phase advance will belong to $S p(2 n) \cap S O(2 n)=U(n)$.

We now present the detailed derivation of the generalized CS theory. We start by quickly reviewing the time-dependent symplectic transformation technique developed by Leach [20]. For a Hamiltonian given by $H_{1}=\frac{1}{2} z_{1}^{T} A_{1}(t) z_{1}$, we seek a time-dependent linear symplectic coordinate transformation $z_{2}=S(t) z_{1}$ such that in the $z_{2}$ coordinates, the dynamics are governed by $H_{2}=\frac{1}{2} z_{2}^{T} A_{2}(t) z_{2}$. Here $H_{1}$ is specified by a $2 n \times 2 n$ symmetric matrix $A_{1}(t)$, and $H_{2}$ is a targeted Hamiltonian specified by a desired $2 n \times 2 n$ symmetric matrix $A_{2}(t)$. The required symplectic matrix $S(t)$ must satisfy the matrix equation [20]

$$
\dot{S}=J A_{2} S-S J A_{1}
$$

where $J=\left(\begin{array}{cc}0 & I \\ -I & 0\end{array}\right)$ is the $2 n \times 2 n$ unit symplectic matrix. The derivation of Eq. (9) can be found in Refs. [16, 17, 20], and its geometric interpretation is given in Ref. [19]. 
We now apply this technique to the Hamiltonian given by Eq. (8). We proceed in two steps. First, we seek a coordinate transformation $\bar{z}=S z$ such that, in the $\bar{z}$ coordinates, the Hamiltonian assumes the form

$$
\bar{H}=\frac{1}{2} \bar{z}^{T} \bar{A} \bar{z}, \bar{A}=\left(\begin{array}{cc}
\mu(t) & 0 \\
0 & \mu(t)
\end{array}\right),
$$

where $\mu(t)$ is a $2 \times 2$ matrix to be determined. In terms of $2 \times 2$ blocks, $S=\left(\begin{array}{cc}S_{1} & S_{2} \\ S_{3} & S_{4}\end{array}\right)$, and Eq. (9) splits into four matrix equations. Including $\mu(t)$, we have five $2 \times 2$ matrices to be determined. Thus we let $S_{2}=0$. We define $w \equiv S_{4}$ because it will be clear later that $S_{4}$ is the envelope matrix. Equation (9) becomes

$$
\begin{aligned}
& \dot{S}_{1}=\mu S_{3}-S_{1} R^{T} \\
& S_{1}=\mu w m, \\
& \dot{S}_{3}=-\mu S_{1}-S_{3} R^{T}+w \kappa, \\
& S_{3}=-\dot{w} m+w R m .
\end{aligned}
$$

Because Eqs. (11)-(14) describe a curve in $S p(4)$, they are consistent with the symplectic condition $S_{1} w^{T}=I$, which implies

$$
\mu=\left(w m w^{T}\right)^{-1}
$$

Then, from Eqs. (13) and (14) we immediately obtain the following matrix differential equation for the envelope matrix $w$,

$$
\frac{d}{d t}\left(\frac{d w}{d t} m-w R m\right)+\frac{d w}{d t} m R^{T}+w\left(\kappa-R m R^{T}\right)-\left(w^{T} w m w^{T}\right)^{-1}=0 .
$$

This is the generalized envelope equation. It generalizes the $1 \mathrm{D}$ envelope equation (4) (or Ermakov-Milne-Pinney equation [21-23]), as well as the previous matrix envelope equation for the case with only quadrupole and skew-quadrupole magnets [16-19]. For $n$-degrees of freedom, the envelope matrix $w$ will be $n \times n$, and the generalized envelope equation has the same format as Eq. (16). Once $w$ is solved for from the envelope equation, we can determine $S_{1}$ from Eq. (12) and $S_{3}$ from Eq. (14). In terms of the envelope matrix $w$, the first symplectic transformation $S$ and its inverse are given by

$$
S=\left(\begin{array}{cc}
w^{-T} & 0 \\
(w R-\dot{w}) m & w
\end{array}\right), S^{-1}=\left(\begin{array}{cc}
w^{T} & 0 \\
\left(w^{-1} \dot{w}-R\right) m w^{T} & w^{-1}
\end{array}\right) .
$$


The second step is to use another coordinate transformation $\overline{\bar{z}}=P(t) \bar{z}$ to transform $\bar{H}$ into a vanishing Hamiltonian $\overline{\bar{H}}=0$, thereby rendering the dynamics trivial in the new coordinates. In this case, the determining equation for the transformation $P(t)$ is

$$
\dot{P}=-P J \bar{A}=P\left(\begin{array}{cc}
0 & -\mu \\
\mu & 0
\end{array}\right)
$$

It is straightforward to verify that $-J \bar{A} \in \operatorname{sp}(4) \bigcap s o(4)$, where $s p(4)$ and $s o(4)$ are the Lie algebras of $S p(4)$ and $S O(4)$, respectively. Thus $P(t)$ is a curve in the group of $4 \mathrm{D}$ symplectic rotations, i.e., $P(t) \in S p(4) \cap S O(4)=U(2)$, provided the initial condition of $P(t)$ is chosen such that $P(0) \in S p(4) \bigcap S O(4)=U(2)$. We call $P(t)$ the generalized phase advance, an appropriate title in light of the fact that $P(t)$ is a symplectic rotation. The Lie algebra element (infinitesimal generator) $-J \bar{A}=\left(\begin{array}{cc}0 & -\mu \\ \mu & 0\end{array}\right)$ is therefore the phase advance rate, and it is determined by the envelope matrix through Eq. (15). Since $S p(4) \cap S O(4)=U(2)$, $P$ and its inverse must have the form of

$$
P=\left(\begin{array}{cc}
P_{1} & P_{2} \\
-P_{2} & P_{1}
\end{array}\right), P^{-1}=P^{T}=\left(\begin{array}{cc}
P_{1}^{T} & -P_{2}^{T} \\
P_{2}^{T} & P_{1}^{T}
\end{array}\right)
$$

Combining the two symplectic coordinate transformations, we obtain the transformation

$$
\overline{\bar{z}}=G(t) z=P(t) S(t) z
$$

As mentioned above, the decomposition of $G$ as a product of a symplectic rotation (unitary) matrix $P$ and a lower-triangular matrix $S$ of the specified form is the well-known Iwasawa decomposition for a semi-simple Lie group [29]. Given that we have derived the decomposition from purely dynamical considerations, the theory developed here can be viewed as a dynamical interpretation of the Iwasawa decomposition for the symplectic group.

Because $\overline{\bar{H}}=0, \overline{\bar{z}}=$ const. Therefore, the symplectic matrix specifying the map between $z_{0}$ and $z=M(t) z_{0}$ is

$$
M(t)=S^{-1} P^{-1} P_{0} S_{0}=\left(\begin{array}{cc}
w^{T} & 0 \\
\left(w^{-1} \dot{w}-R\right) m w^{T} & w^{-1}
\end{array}\right) P^{T}\left(\begin{array}{cc}
w^{-T} & 0 \\
(w R-\dot{w}) m & w
\end{array}\right)_{0},
$$

where subscript " 0 " denotes initial conditions at $t=0$, and $P_{0}$ is taken to be $I$ without lose of generality since $P$ is a symplectic rotation. This expression for $M(t)$ generalizes 
the decomposition of the symplectic map for the original 1D CS theory given by Eq. (5). Specifically, the 2D rotation matrix, which is also symplectic, in Eq. (5) is generalized into a 4D symplectic rotation $P^{T}$. The phase advance $P$ is generated by the infinitesimal generator determined by the envelope matrix through $\mu=\left(w m w^{T}\right)^{-1}$, just as in the original 1D CS theory where the infinitesimal generator of the phase advance is $w^{-2}$ for a scalar envelope $w$. The first and the third matrices in Eq. (5) obviously have the same construction as their counterparts in Eq. (21).

Given the lattice function $A(t)$, we need to solve the envelope equation (16) and the phase advance equation (18) to construct $M(t)$ according to Eq. (21). The initial conditions for $w$ and $\dot{w}$ can be arbitrary except that they have to satisfy the symplectic condition $w S_{3}^{T}=S_{3} w^{T}$, which is a single constraint since $w$ is a $2 \times 2$ matrix. The envelope equation (16) admits a $1 \mathrm{D}$ gauge freedom as well. For any constant matrix $c \in O(2)$, we can show by straightforward calculation that if $w$ is a solution of Eq. (16), then $c w$ is also a solution. However, $P(t)$ and $M(t)$ are independent of this gauge. The number of initial conditions for Eq. (16) is 8, and the dimension of $P(t)$ as the $U(2)$ group is 4 . Subtracting the one symplectic constraint on the initial conditions and the one gauge freedom, the total number of independent parameters for $M(t)$ is 10 , as expected.

Because $\overline{\bar{z}}=$ const,

$$
I_{\xi}=z^{T} S^{T} P^{T} \xi P S z
$$

is a constant of motion for any constant $4 \times 4$ positive definite matrix $\xi$. This expression generalizes the CS invariant [1] (or Lewis invariant [24, 25]) for 1-degree of freedom in Eq. (6). In particular, we can define $I_{\xi}$, with $\xi$ being the unit matrix, to be the special invariant corresponding to the original CS invariant. In this case, the phase advance $P$ in Eq. (22) drops out, and

$$
I_{C S} \equiv z^{T} S^{T} S z=z^{T}\left(\begin{array}{cc}
\gamma & \alpha \\
\alpha^{T} & \beta
\end{array}\right) z,
$$

where $\alpha, \beta$, and $\gamma$ are the generalized Twiss parameters defined by

$$
\begin{aligned}
& \alpha \equiv w^{T} S_{3}, \\
& \beta \equiv w^{T} w \\
& \gamma \equiv S_{3}^{T} S_{3}+w^{-1} w^{-T} .
\end{aligned}
$$


It is straightforward to verify that as time-dependent $2 \times 2$ matrices, $\alpha, \beta$, and $\gamma$ satisfy

$$
\beta \gamma=I+\alpha^{2}
$$

which is a familiar relationship in the original CS theory between the scalar Twiss parameters defined by Eqs. (3) and (7). Use has been made of the symplectic condition, $w S_{3}^{T}=S_{3} w^{T}$, in obtaining Eq. (23).

The envelope matrix $w$ and the invariant $I_{\xi}$ define the beam dimensions and emittance for both low intensity beams and high intensity beams with strong space-charge potential. Investigation of these properties will be reported on in future publications. Here we demonstrate how to use the generalized theory to study the instability properties of the transverse dynamics governed by the Hamiltonian $H(t)$ specified in Eq. (8) for a general periodic lattice. After solving for a matched envelope matrix, the one-turn map $M_{c}(t)$ is given by

$$
M_{c}(t)=S^{-1}(t) P_{c}^{T}(t) S(t)
$$

where $P_{c}^{T}(t)$ is the one-turn phase advance transposed. Equation (24) states that $M_{c}(t)$ is similar to $P_{c}^{T}(t)$, and thus we reach the remarkable conclusion that the stability properties of the dynamics are completely determined by the one-turn phase advance $P_{c}(t)$. This significantly simplifies the stability analysis and showcases the physical relevance of the generalized CS parameterization scheme developed here. Since $P_{c}(t)$ is a symplectic rotation, whose determinant is one, we obtain the following stability criterion: a necessary and sufficient condition for the general transverse dynamics to be unstable is that $P_{T}(t)$ has an eigen value $\lambda$ with $|\lambda| \neq 1$.

This research was supported by the U.S. Department of Energy (DE-AC02-09CH111466 and DE-AC02-07CH11359).

[1] E. Courant and H. Snyder, Annals of Physics 3, 1 (1958).

[2] S. Y. Lee, Accelerator Physics (World Scientific, Singapore, 1999), p. 47.

[3] H. Qin and R. C. Davidson, Physical Review Special Topics - Accelerators and Beams 9, 054001 (2006). 
[4] I. Kapchinskij and V. Vladimirskij, in Proc. of the International Conference on High Energy Accelerators and Instrumentation (CERN Scientific Information Service, Geneva, 1959), p. 274.

[5] H. Qin, M. Chung, and R. C. Davidson, Physical Review Letters 103, 224802 (2009).

[6] H. Qin and R. C. Davidson, Physical Review Letters 110, 064803 (2013).

[7] A. Friedman, J. J. Barnard, R. H. Cohen, D. P. Grote, S. M. Lund, W. M. Sharp, A. Faltens, E. Henestroza, J.-Y. Jung, J. W. Kwan, et al., Physics of Plasmas 17, 056704 (2010).

[8] L. Michelotti, Intermediate classical dynamics with applications to beam physics (John Wiley \& Sons Inc., 1995), chap. 3, p. 166.

[9] L. C. Teng, NAL Report FN-229 (1971).

[10] D. A. Edwards and L. C. Teng, IEEE Trans. Nucl. Sci. NS-20, 885 (1973).

[11] L. C. Teng, in Proceedings of the 2003 Particle Accelerator Conference (Piscataway, NJ, 2003), p. 2895 .

[12] H. Grote, F. Iselin, E. Keil, and J. Niederer, in Proceedings of 1989 Particle Accelerator Conference (IEEE, Piscataway, NJ, 1989), p. 1292.

[13] G. Dattoli, C. Mari, M. Richetta, and A. Torre, Nuovo Cimento 107B, 269 (1992).

[14] G. Ripken, Tech. Rep. R1-70/04, DESY (1970).

[15] H. Wiedemann, Particle Accelerator Physics (Springer-Verlag, 2007), pp. 614-620.

[16] H. Qin and R. C. Davidson, Physical Review Special Topic - Accelerators and Beams 12, 064001 (2009).

[17] H. Qin and R. C. Davidson, Phys. Plasmas 16, 050705 (2009).

[18] M. Chung, H. Qin, and R. C. Davidson, Physics of Plasmas 17, 084502 (2010).

[19] H. Qin and R. C. Davidson, Physics of Plasmas 18, 056708 (2011).

[20] P. Leach, Journal of Mathematical Physics 18, 1608 (1977).

[21] V. Ermakov, Univ. Izv. Kiev 20, 1 (1880).

[22] W. E. Milne, Physical Review 35, 863 (1930).

[23] E. Pinney, Proceedings of the American Mathematical Society 1, 681 (1950).

[24] H. R. Lewis, Journal of Mathematical Physics 9, 1976 (1968).

[25] H. R. Lewis and W. B. Riesenfeld, Journal of Mathematical Physics 10, 1458 (1969).

[26] Berry, Journal of Physics A: Mathematical and General 18, 15 (1985).

[27] D. A. Morales, Journal of Physics A: Mathematical and General 21, L889 (1988). 
[28] D. B. Monteoliva, H. J. Korsch, and J. A. Nunez, Journal of Physics A: Mathematical and General 27, 6897 (1994).

[29] K. Iwasawa, Annals of Mathematics 50, 507 (1949). 